\title{
Stochastic Optimal Sizing of Micro-Grids Using the Moth-Flame Optimization Algorithm
}

\author{
Soheil Mohseni, Alan C. Brent, Daniel Burmester, Abhi Chatterjee \\ Sustainable Energy Systems, School of Engineering and Computer Science, Victoria University of Wellington, New Zealand \\ Email: \{soheil.mohseni, abhi.chatterjee\}@ecs.vuw.ac.nz, \{alan.brent, daniel.burmester\}@vuw.ac.nz
}

\begin{abstract}
Optimal sizing of renewable and sustainable energy systems should consider the uncertainties associated with various input data to ensure the financial sustainability of developing such systems, especially in the case of stand-alone systems. This paper proposes a novel stochastic modelling framework for the optimal sizing of micro-grids subject to satisfying a reliability index for supplying the loads. The proposed framework incorporates a model reduction technique, a state-of-the-art meta-heuristic optimization algorithm (i.e. moth-flame optimization algorithm), as well as an uncertainty analysis technique using Monte Carlo simulations based on a new scenario reduction process. It also preserves the computational tractability. A micro-grid test system incorporating photovoltaic panels, wind turbines, battery packs, a $\mathrm{DC} / \mathrm{AC}$ inverter, and an electric vehicle fast charging station is used to assess the validity and effectiveness of the proposed stochastic framework. Accordingly, the impact of uncertainties associated with renewable power generation and load demand on the sizes of the considered micro-grid components are evaluated. The numerical simulation results for the considered micro-grid test system are presented and compared with those generated by a deterministic model, which have demonstrated the effectiveness of the proposed stochastic modelling framework.
\end{abstract}

Index Terms--Distributed power generation, Microgrids, Power system planning, Power system reliability, Smart grids.

\section{INTRODUCTION}

A micro-grid is defined as a distinct, small-scale, smart power grid, which provides a platform for the efficient integration of distributed energy resources (DER) and various types of loads within clearly defined electrical borders that acts as a single controllable entity [1]. Micro-grids can lay the foundations for taking advantage of renewable energy sources (RES) to reduce greenhouse gas (GHG) emissions and provide electricity access to remote communities, diversifying electricity supply, and driving economic growth [2].

Optimal planning of micro-grids is vital as this ensures the economic viability of such systems, whilst adhering to constraints in terms of power supply reliability and system operation. As a particular problem in the planning phase of micro-grids, optimal sizing of the components of micro-grids is of the highest importance as it calculates the optimum combination for the sizes of components and a seemingly small change in the number/capacity of each component, in effect, considerably impacts the total cost of the system [3].

The optimal sizing problem of the components of microgrids is a non-deterministic polynomial-time hard (NP-hard) problem with several sources of nonlinearities and nonconvexities involved in its formulation, making it not amenable to the exact mathematical optimization algorithms [4]. Therefore, several meta-heuristic optimization algorithms have been proposed in the literature to solve this problem, among which the particle swarm optimization (PSO) and genetic algorithm (GA) are the most preferred approaches in the area, while the hybrid GA-PSO is the most recent one, which is proved to be superior to the GA and PSO [5]. Nonetheless, applying the meta-heuristics to the considered sizing problems remains an open research area as it is always possible that a newly emerged meta-heuristic outperforms those currently applied, especially since according to the no free lunch theorem [6], no meta-heuristic optimization algorithm is most suited for solving all optimization problems.

The other issue that exacerbates the problem of nearing the globally optimum solutions, while calculating the sizes of micro-grid components, is the uncertainty associated with various input data. Among the uncertain input data, the climatic and load demand have the most significant influence on the results and modelling their uncertainty in the optimal sizing procedure is a big step toward an accurate representation of real-world scenarios through numeric simulations [7].

In recent years there has been growing interest in considering the uncertainties, while optimally designing the renewable energy systems. Various approaches have also been proposed to model the uncertainties in the planning phase of such systems, among which the stochastic simulation of the uncertain parameters using the Monte Carlo simulation (MCS) method is the most-favoured one in the literature [7]. This is because of the simplicity and general applicability of the MCS method, as well as its ability to cope with little knowledge about the historical data. Despite the important advantages of the MCS method, it is usually slow. This has prohibited researchers from increasing the number of uncertain parameters within their stochastic models, when using the MCS method for handling the uncertain parameters and made them neglect the less important sources of uncertainty within their systems [8]. The main contributions of this paper are summarized below.

- The effectiveness of the moth-flame optimization algorithm (MFOA) [9] in solving the optimal sizing problem of microgrids is investigated.

- A novel stochastic modelling framework is proposed, which provides the capability of considering the effects of uncertain input data in the micro-grid optimal sizing studies.

- The impact levels of some uncertain input variables on the lifetime cost variability of micro-grids are evaluated through conducting sensitivity analyses. 


\section{SYSTEM MODELLING}

This section presents the components, configuration, and power flow of a stand-alone micro-grid, which is used as a test system to validate the effectiveness of the proposed stochastic modelling framework for optimal sizing of micro-grids.

A schematic representation of the proposed off-grid microgrid test system is presented in Fig. 1. The proposed micro-grid test system in this study consists of photovoltaic (PV) panels, wind turbines (WTs), a battery bank, some converters, and an electric vehicle (EV) fast charging station, which are described in detail below.

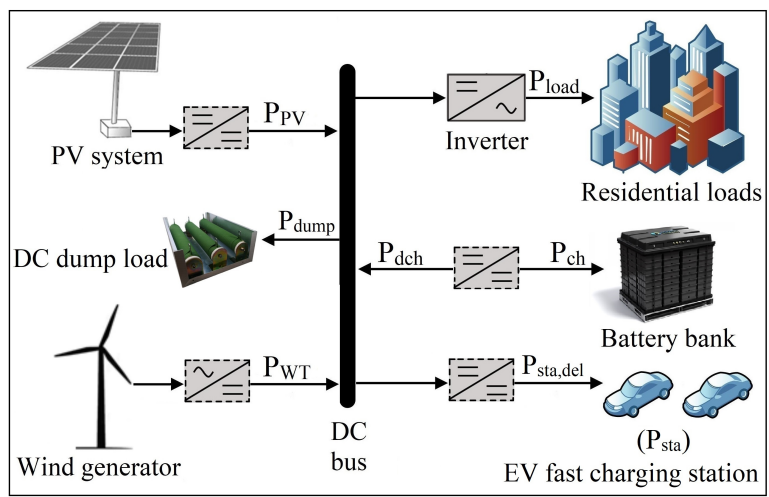

Fig. 1. Schematic diagram of the proposed micro-grid test system.

\section{A. Description of the Micro-Grid Components}

The total output power of $325-\mathrm{W}$ PV panels at time step $t$ can be calculated by Eq. (1) [5].

$$
P_{P V}(t)=N_{P V} \times \eta_{g} \times A_{m} \times \frac{G_{t}(t)}{1000},
$$

where $N_{P V}$ is the number of panels, $\eta_{g}$ is the efficiency of PV panels, i.e. $16.9 \%$ [10], $A_{m}$ is the area of each panel used in the micro-grid system $\left[\mathrm{m}^{2}\right]$, i.e. $1.92 \mathrm{~m}^{2}[10]$, and $G_{t}(t)$ is the total solar irradiance incident on the panels $\left[\mathrm{W} / \mathrm{m}^{2}\right]$ at time step $t$.

The output power of the WTs at time step $t$ is described in terms of wind speed in Eq. (2) [11].

$$
P_{W T}(t)=N_{W T} \times \begin{cases}0 & ; V<V_{\text {cin }}, V>V_{\text {cout }} \\ P_{r} \times\left(\frac{V(t)-V_{\text {cin }}}{V_{r}-V_{\text {cin }}}\right)^{3} & ; V_{\text {cin }} \leq V<V_{r} \\ P_{r} & ; V_{r} \leq V \leq V_{\text {cout }}\end{cases}
$$

where $V_{\text {cin }}, V_{\text {cout }}$, and $V_{r}$ are the cut-in, cut-out, and rated wind speeds, respectively that are $2.7,20$, and $7.5 \mathrm{~m} / \mathrm{s}$, respectively; $V(t)$ is the wind speed at time step $t ; N_{W T}$ is the number of WTs; and $P_{r}$ is the nominal power of each WT, i.e. $10 \mathrm{~kW} \mathrm{[10].}$

The DC fast charging electric vehicle supply equipment (EVSE) is considered in this study to charge the batteries of EVs that arrive at the station. The efficiency of each fast charger is denoted by $\eta_{s t a}$, i.e. $85 \%$. The rated power of each charger is $50 \mathrm{~kW}$ that can be operated with the DC voltage of 400-500 VDC and accepts the maximum current of 100-125 A [12].

The energy stored in the battery bank, consisted of 1,156-Ah battery packs, at time step $t$ can be expressed by Eq. (3) [13].

$$
E_{b a t}(t)=E_{b a t}(t-1)+P_{c h}(t) \times \eta_{b a t} \times \Delta t-\left(\frac{P_{d c h}(t)}{\eta_{b a t}}\right) \times \Delta t,(3)
$$

where $P_{c h}$ is the transferred power from the RES to the battery packs, $P_{d c h}$ is the transferred power from the battery packs to the DC/AC inverter, and $\eta_{b a t}$ represents both the charge and discharge efficiencies of the battery bank, i.e. $85 \%$ [13].

Furthermore, the maximum allowable depth of discharge (DOD) of the battery bank is considered to be 0.8 [13].

The DC input of the inverter, considered to serve the AC residential loads on the micro-grid, can be from the DC output of the WTs, PV panels, and battery bank. The efficiency of the inverter is denoted by $\eta_{\text {inv }}$, which is considered to be $90 \%$ [14].

A three-phase AC/DC converter is used to couple the WTs to the common DC bus of the micro-grid network, while this is performed through two DC/DC converters for the PV panels and EVSE, as well as a bi-directional DC/DC converter for the battery bank. The efficiencies and costs associated with these converters are considered in their respective components, and hence they are shown inside the dashed lines in Fig. 1.

\section{B. Operational Strategy}

\section{1) Generation Meets Demand}

In this situation, the power generated by PV arrays and WTs is equal to the sum of residential and $\mathrm{EV}$ charging loads; hence:

$$
\begin{gathered}
P_{P V}(t)+P_{W T}(t)=\left(P_{\text {load }}(t) / \eta_{\text {inv }}\right)+\left(P_{\text {sta }}(t) / \eta_{\text {sta }}\right), \\
E_{\text {bat }}(t+\Delta t)=E_{\text {bat }}(t), \\
P_{\text {sta }, \text { del }}(t)=P_{\text {sta }}(t) .
\end{gathered}
$$

\section{2) Over Generation}

If the generated renewable power is more than the power requirements of the residential and $\mathrm{EV}$ charging loads, the excess power will be used for charging of battery packs; hence:

$$
\begin{gathered}
P_{c h}(t)=P_{P V}(t)+P_{W T}(t)-\left(P_{\text {load }}(t) / \eta_{\text {inv }}\right)-\left(P_{\text {sta }}(t) / \eta_{\text {sta }}\right), \\
E_{\text {bat }}(t+\Delta t)=E_{\text {bat }}(t)+P_{c h}(t) \times \eta_{c h} \times \Delta t, \\
P_{\text {sta,del }}(t)=P_{\text {sta }}(t) .
\end{gathered}
$$

\section{3) Over Demand}

If there is a shortage of power generation for supplying the residential loads, the battery bank will be discharged to compensate the shortage of power generation; hence:

$$
\begin{gathered}
P_{d c}(t)=\left(P_{\text {load }}(t) / \eta_{\text {inv }}\right)-P_{P V}(t)-P_{W T}(t), \\
E_{\text {bat }}(t+\Delta t)=E_{\text {bat }}(t)-\left(P_{d c h}(t) / \eta_{d c}\right) \times \Delta t, \\
P_{\text {sta,del }}(t)=0 .
\end{gathered}
$$

In this situation, if the delivered power to the battery bank exceeds its rated power, the surplus power generated by the RES will be dissipated by a DC dump load, the cost of which is considered to be negligible.

Furthermore, the time step increment $(\Delta t)$ is considered to be 1 hour in all the above situations and it is assumed that the energy stored in the battery bank is not delivered to the charging station to avoid energy losses caused by energy conversion.

\section{PROBLEM FormulATION}

This section presents the mathematical formulation of the proposed modelling framework developed for optimal sizing of the considered micro-grid test system.

\section{A. Model Reduction}

According to the computationally intensive nature of the 
considered problem, motivated by [15], the one-year hourly generation and load data are reduced to 288 data points per data stream by deriving their monthly averaged daily profiles.

\section{B. Objective Function}

The net present cost (NPC) method is used in this study to calculate the whole-life cost of the system according to the procedure suggested in [11]. The NPC of each micro-grid component consists of its capital, replacement, and operation and maintenance $(\mathrm{O} \& \mathrm{M})$ costs, minus a salvage value if applicable. The sum of NPCs of the micro-grid components is selected as the objective function, which can be expressed by Eq. (7). The project lifetime and real discount rate are considered to be 20 years and $6 \%$, respectively.

$$
N P C=N P C_{P V}+N P C_{W T}+N P C_{b a t}+N P C_{i n v}+N P C_{E V S E} .
$$

\section{Reliability Assessment}

The equivalent loss factor (ELF) reliability metric is considered in this paper for reliability assessment of the microgrid, which can be defined as in Eq. (8) [16].

$$
E L F=\frac{1}{T} \sum_{t=1}^{T} \frac{Q(t)}{P(t)},
$$

where $P(t)$ and $Q(t)$ are the total load demand and loss of load at time step $t$, respectively; and $T$ denotes the operational time frame, i.e. 288 hours in compliance with the reduced model.

\section{Constraints}

The objective function defined by Eq. (7) must be minimized subject to the constraints in Eqs. (9)-(13).

$$
\begin{gathered}
E L F \leq 0.01, \\
E_{\text {bat,min }} \leq E_{\text {bat }}(t) \leq E_{\text {bat } \text { max }}, \quad \forall t \in\{1, \ldots, T\} \\
E_{\text {bat }}(t) \geq(1-D O D) \times E_{\text {bat,max }}, \quad \forall t \in\{1, \ldots, T\} \\
E_{\text {bat }}(288) \geq E_{\text {bat }}(0), \\
\sum_{t=1}^{288} P_{\text {dump }}(t) \leq 0.05 \times \sum_{t=1}^{288}\left(P_{P V}(t)+P_{W T}(t)\right) .
\end{gathered}
$$

\section{E. Optimization Algorithm}

MFOA is a new nature-inspired, meta-heuristic optimization technique, conceptualized from the navigation system of moths, [9]. It starts by creating the moths and flames, which represent the search agents and their best positions found so far, respectively. It then calculates their corresponding fitness values and updates the position of each moth with respect to a flame using a logarithmic spiral function according to Eq. (14).

$$
S\left(M_{i}, F_{j}\right)=D_{i} e^{b r} \cos (2 \pi r)+F_{j},
$$

where $M_{i}$ indicates the $i^{t h}$ moth, $F_{j}$ represents the $j^{\text {th }}$ flame, $D_{i}$ denotes the distance from the $i^{\text {th }}$ moth to the $j^{\text {th }}$ flame, $S$ is the spiral function, $b$ is a constant that defines the shape of the logarithmic spiral, and $r$ is a random number in the range $[-1,1]$.

This procedure is run iteratively until a stopping criterion is met. In this analysis, we assumed $b=1$, a population size of 45 for the search agents, and considered the maximum number of iterations of 300 as the stopping criterion.

\section{F. MCS-Based Stochastic Modelling of Uncertainties}

A combination of the MCS method and the $K$-means clustering algorithm is utilized in this study to model the uncertain input variables. Accordingly, following the compression of relevant data, each time step of the historical solar irradiance, wind speed, and load demand data (that capture their long-term trends), is assigned a Beta, Weibull, and Normal probability density function (PDF), as they have been proven to be the best suited PFDs for the corresponding data stream and is dealt with independently of other time steps [17]. Then, using a stochastic approach, 500 scenario sets are generated through the sampling of the individual PDFs mentioned above. The 500 scenario sets are then reduced to 5 scenario sets using the $K$ means clustering algorithm, which retains the decision-relevant characteristics of the whole scenario sets at an acceptable level, to maintain a tractable computational burden. As reported in [18], the $K$-means clustering algorithm is a practical and efficient approach in reducing the number of scenarios towards mitigating the computational complexity, whilst maintaining an acceptable solution accuracy. The system is then optimized for each 5 scenario sets and the expected values of the simulation results obtained for them are selected as the final solution of the optimal sizing problem considering the aforementioned uncertainties.

\section{G. A Panorama of Optimization Procedure}

The flowchart of the proposed stochastic modelling framework for optimal sizing of micro-grids is shown in Fig. 2.

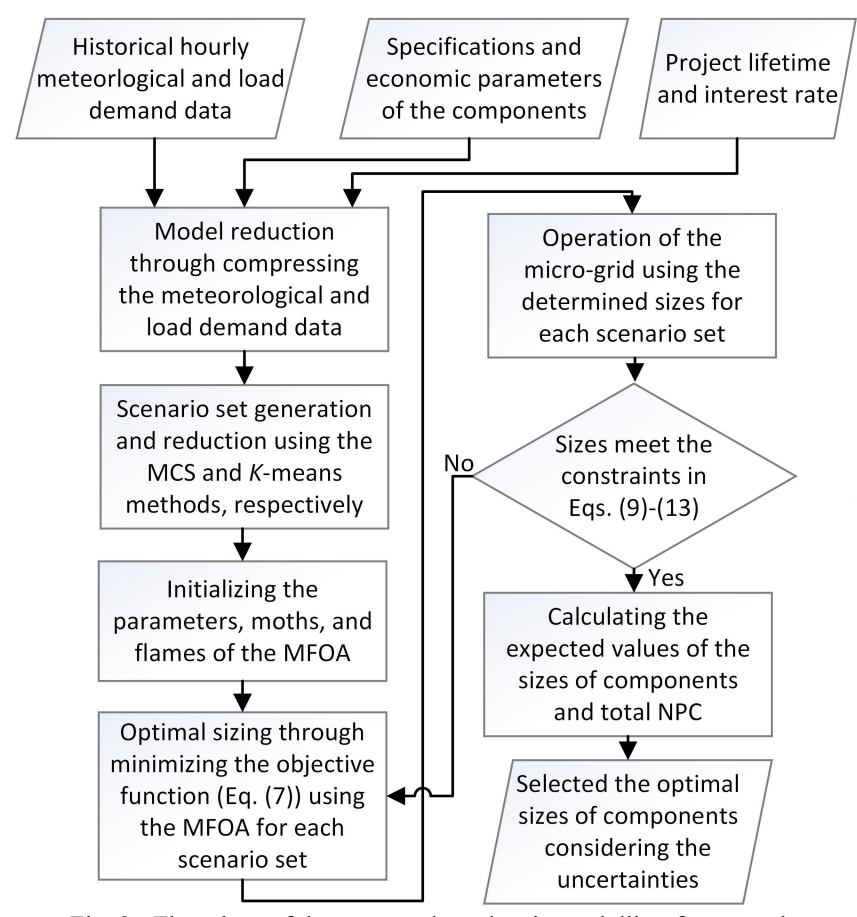

Fig. 2. Flowchart of the proposed stochastic modelling framework.

\section{Simulation Results AND Discussion}

The islanded micro-grid test system shown in Fig. 1 is used to evaluate the effectiveness and validity of the proposed stochastic modelling framework through numeric simulations using MATLAB. 


\section{A. Input Data}

A case study was undertaken to optimally size the considered conceptual micro-grid for Hengam Island in the Persian Gulf, Iran (latitude $26.6536^{\circ} \mathrm{N}$, longitude $55.8802^{\circ} \mathrm{E}$ ).

The solar irradiance and wind speed data for the considered site were obtained from the Iran Meteorological Organization in a one-year hourly average basis for the period 2007-2016, which are reduced by the considered model reduction technique and their monthly averaged 24 -h profiles are shown in Fig. 3. The forecasted monthly averaged $24-\mathrm{h}$ color plot of the total load on the hypothetical micro-grid is also shown in Fig. 4.

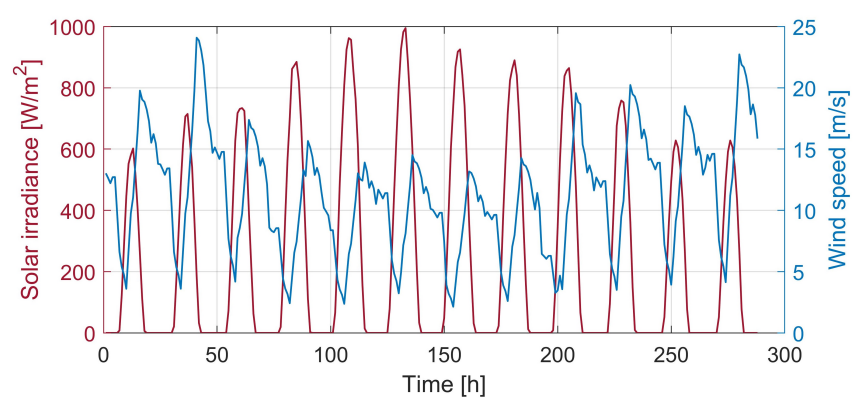

Fig. 3. Monthly averaged daily profiles for the climatic data.

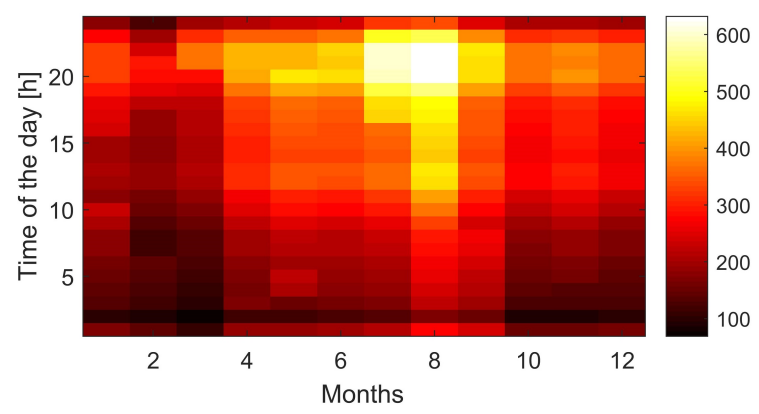

Fig. 4. Monthly averaged daily variation of the load on the micro-grid [kW].

The product model and specifications of the components of the micro-grid are shown in Table I [10], [12], [14], [19].

\section{B. Verification of the MFOA}

The best solution sets of the results obtained by solving the considered optimal sizing problem using the MFOA, GA, PSO, and hybrid GA-PSO in the context of the proposed modelling framework with the same number of search agents from 20 independent runs, are shown in Table II. The total NPC (TNPC) of the micro-grid in terms of iterations for the aforementioned optimization algorithms, averaged over 20 runs is also shown in Fig. 5. The results demonstrate that the MFOA outperforms the GA and PSO (i.e. the most preferred meta-heuristics applied in this research area), as well as the hybrid GA-PSO (i.e. the most recent one), in terms of nearing the optimal solutions (i.e. optimization accuracy). Also, the number of trials that confirmed the supremacy of the MFOA is appreciable, ensuring the repeatability and reproducibility of the results (i.e. optimization precision).

Notice that as an implication of the no free lunch theorem [6], identifying the best suited stochastic optimization algorithm to solve the optimal capacity planning problems of the sustainable energy systems is a continuously developing field. Hence, no definitive conclusions can be made regarding the appropriateness of a meta-heuristic optimization in this research context without employing it to solve at least one representative micro-grid capacity optimization problem and evaluating its efficiency compared to the well-known and established algorithms via a trial-and-error mechanism.

TABLE I

Specs of the micro-grid components [10], [12], [14], [19]

\begin{tabular}{|c|c|c|c|c|c|}
\hline Component & $\begin{array}{c}\text { Product } \\
\text { model }\end{array}$ & $\begin{array}{c}\text { Capital } \\
\text { cost }\end{array}$ & $\begin{array}{l}\text { Replace- } \\
\text { ment cost }\end{array}$ & $\begin{array}{c}\mathrm{O} \& \mathrm{M} \\
\text { cost }\end{array}$ & $\begin{array}{c}\text { Lifetime } \\
\text { [year] }\end{array}$ \\
\hline PV panel & $\begin{array}{l}\text { CS6U- } \\
325 \mathrm{P}\end{array}$ & $\begin{array}{l}\$ 700 \\
\text { /unit }\end{array}$ & $\begin{array}{l}\$ 500 \\
\text { /unit }\end{array}$ & $\begin{array}{c}\$ 14 / \\
\text { unit/yr }\end{array}$ & 20 \\
\hline $\begin{array}{l}\text { Wind } \\
\text { turbine }\end{array}$ & EO10 & $\begin{array}{c}\$ 30000 \\
\text { /unit }\end{array}$ & $\begin{array}{c}\$ 30000 \\
\text { /unit }\end{array}$ & $\begin{array}{l}\$ 600 / \\
\text { unit/yr }\end{array}$ & 20 \\
\hline $\begin{array}{c}\text { Battery } \\
\text { bank }\end{array}$ & $\begin{array}{c}\text { 6-CS- } \\
25 \mathrm{P}\end{array}$ & $\begin{array}{c}\$ 1229 \\
\text { /unit }\end{array}$ & $\begin{array}{c}\$ 1229 \\
\text { /unit }\end{array}$ & $\begin{array}{c}\$ 10 / \\
\text { unit/yr }\end{array}$ & 10 \\
\hline $\begin{array}{l}\mathrm{DC} / \mathrm{AC} \\
\text { inverter }\end{array}$ & $\begin{array}{l}\text { GTP- } \\
519 \mathrm{~S}\end{array}$ & $\begin{array}{l}\$ 300 \\
/ \mathrm{kW}\end{array}$ & $\begin{array}{l}\$ 300 \\
/ \mathrm{kW}\end{array}$ & $\begin{array}{c}\$ 10 / \\
\mathrm{kW} / \mathrm{yr}\end{array}$ & 10 \\
\hline $\begin{array}{l}\text { EV fast } \\
\text { charger }\end{array}$ & $\begin{array}{c}\text { Generic } \\
50 \mathrm{~kW}\end{array}$ & $\begin{array}{c}\$ 6000 \\
\text { /unit }\end{array}$ & $\begin{array}{l}\$ 6000 \\
\text { /unit }\end{array}$ & $\begin{array}{c}\$ 25 / \\
\text { unit/yr }\end{array}$ & 20 \\
\hline
\end{tabular}

TABLE II

Comparison of the best performances of the algorithms

\begin{tabular}{ccccccc}
\hline Algorithm & WTs & $\begin{array}{c}\text { PV } \\
\text { panels }\end{array}$ & $\begin{array}{c}\text { Battery } \\
\text { packs }\end{array}$ & $\begin{array}{c}\text { Inverter } \\
{[\mathrm{kW}]}\end{array}$ & EVSE & $\begin{array}{c}\text { TNPC } \\
{[\$]}\end{array}$ \\
\hline MFOA & 45 & 686 & 59 & 338 & 3 & $4,509,119$ \\
\hline GA-PSO & 45 & 689 & 63 & 342 & 4 & $4,522,083$ \\
\hline GA & 46 & 686 & 68 & 349 & 4 & $4,638,701$ \\
\hline PSO & 46 & 689 & 81 & 340 & 4 & $4,601,573$ \\
\hline
\end{tabular}

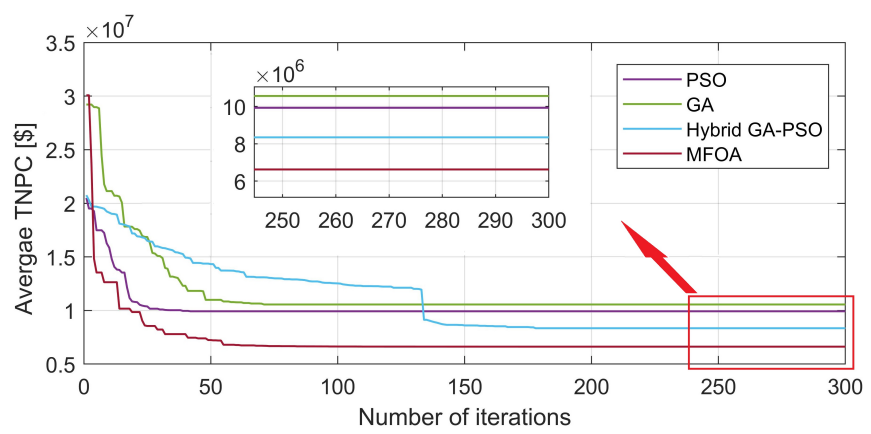

Fig. 5. The average values of TNPC over 20 runs in terms of iterations.

\section{Effects of Uncertainties}

In order to assess the effects of considering the uncertainties associated with the total electrical load and climatic data (as the most influencing uncertainties within the considered micro-grid test system), the results obtained by solving the optimal sizing problem are compared in Table III for the stochastic and deterministic cases. The stochastic results are obtained by applying the stochastic modelling framework developed in Section III, while the deterministic case ignores the stochasticity of the model and considers the climatic and load demand data as certain input variables.

TABLE III

Comparison of deterministic vs. average stochastic results

\begin{tabular}{ccccccc}
\hline Case & WTs & $\begin{array}{c}\text { PV } \\
\text { panels }\end{array}$ & $\begin{array}{c}\text { Battery } \\
\text { packs }\end{array}$ & $\begin{array}{c}\text { Inverter } \\
{[\mathrm{kW}]}\end{array}$ & $\begin{array}{c}\text { EV } \\
\text { SE }\end{array}$ & $\begin{array}{c}\text { TNPC } \\
{[\$]}\end{array}$ \\
\hline Stochastic & 51 & 699 & 70 & 351 & 3 & $4,821,491$ \\
\hline Deterministic & 45 & 686 & 59 & 338 & 3 & $4,509,119$ \\
\hline
\end{tabular}


It can be observed from Table III that considering the aforementioned uncertainties has contributed to increasing the sizes of all the components except the level 3 fast chargers, which in turn has increased the TNPC of the micro-grid by circa $7 \%$. In fact, this extra cost imposed on the system is associated with providing the robustness against the studied uncertainties. This result is significant at the planning stage and would seem to demonstrate that handling the uncertainties associated with renewable power generation and load demand is crucial, while optimally designing the renewable and sustainable energy systems, and if neglected, could slow down the proliferation of such systems in the medium to long-term perspectives.

\section{Sensitivity Analyses}

Several sensitivity analyses are conducted to examine the sensitivity of the TNPC of the micro-grid to variations in uncertain variables of the system, which are carried out by varying the climatic and load demand data from $50 \%$ to $150 \%$ within $10 \%$ intervals, and then optimizing the system using the MFOA in the context of the developed model. The results obtained from the sensitivity analyses are shown in Fig. 6. Comparison of the results of the detailed sensitivity analyses indicates the dominance of the total load demand, and the relatively less importance of the climatic data. Furthermore, it can be seen from Fig. 6 that the wind speed has a greater influence on the TNPC, compared with the solar irradiance, owing to the higher share of WTs in total power generation compared with the PV panels within the micro-grid test system.

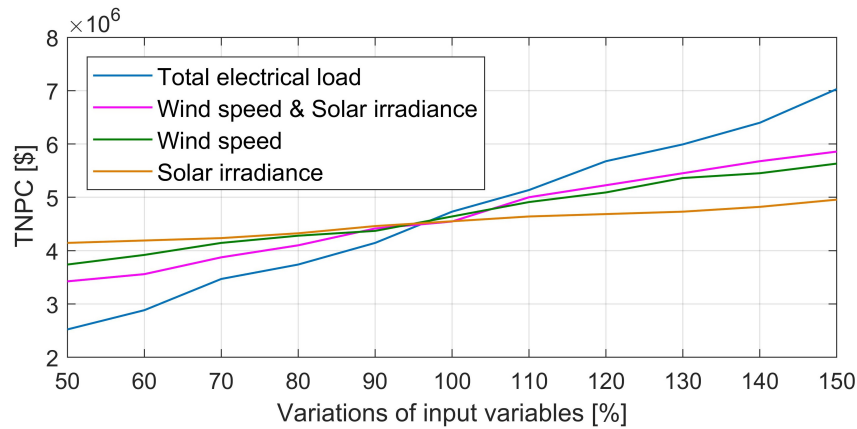

Fig. 6. Sensitivity analyses of the uncertain variables vs. the TNPC.

\section{CONCLUSIONS}

This paper has devised a cutting-edge modelling framework for the optimal sizing of micro-grids under uncertainties, whilst attempting to underline the usefulness of the MFOA in improving the quality of solutions in micro-grid optimal sizing studies. The results of this study indicate that considering the uncertain input variables is vital in the planning phase of sustainable energy systems so that the actual cost-effective combination of the sizes of components does not vary from the optimal solution calculated through numerical simulations. Our research has also stressed the importance of sensitivity analyses in order to offer a better understanding of system uncertainties to the system operators, thus enabling them better to cope with uncertainties. The proposed stochastic modelling framework can be easily extended and adapted to other sources of uncertainty, as well as other case studies. Accordingly, other uncertain parameters can be incorporated into the model, given that the PDFs that best fit their historical data are known. Moreover, combining the $K$-means technique with the MC simulations, along with reducing the model through data compression have paved the way for taking into account several uncertainties at the design phase of renewable energy systems.

\section{REFERENCES}

[1] D. T. Ton and M. A. Smith, "The U.S. Department of Energy's Microgrid Initiative," Electr. J., vol. 25, no. 8, pp. 84-94, Oct. 2012.

[2] G. Liu, Z. Li, M. Starke, T. B. Ollis, and K. Tomsovic, "Optimal sizing of energy storage for community microgrids considering building thermal dynamics," in Proc. IEEE Power Energy Soc. Gen. Meet., 2017, Chicago, IL, USA, pp. 1-5.

[3] S. Mohseni and A. Brent, "Smart grid and zero-emissions energy systems: The need for a multi-dimensional investment planning perspective," IEEE Smart Grid Newsl., vol. A Special Issue on the Paris Climate Agreement and the Role of the Smart Grid, Jun. 2018.

[4] A. Maleki and F. Pourfayaz, "Optimal sizing of autonomous hybrid photovoltaic/wind/battery power system with LPSP technology by using evolutionary algorithms," Sol. Energy, vol. 115, pp. 471-483, May 2015.

[5] N. Ghorbani, A. Kasaeian, A. Toopshekan, L. Bahrami, and A. Maghami, "Optimizing a hybrid wind-PV-battery system using GA-PSO and MOPSO for reducing cost and increasing reliability," Energy, vol. 154, pp. 581-591, Jul. 2018.

[6] D. H. Wolpert and W. G. Macready, "No free lunch theorems for optimization," IEEE Trans. Evol. Comput., vol. 1, pp. 67-82, Apr. 1997.

[7] G. Mavromatidis, K. Orehounig, and J. Carmeliet, "A review of uncertainty characterisation approaches for the optimal design of distributed energy systems," Renew. Sustain. Energy Rev., vol. 88, pp. 258-277, May 2018.

[8] S. Nojavan, M. Majidi, and N. N. Esfetanaj, "An efficient cost-reliability optimization model for optimal siting and sizing of energy storage system in a microgrid in the presence of responsible load management," Energy, vol. 139, pp. 89-97, Nov. 2017.

[9] S. Mirjalili, "Moth-flame optimization algorithm: A novel nature-inspired heuristic paradigm," Knowledge-Based Syst., vol. 89, pp. 228-249, Nov. 2015.

[10] A. C. Duman and Ö. Güler, "Techno-economic analysis of off-grid $\mathrm{PV} /$ wind/fuel cell hybrid system combinations with a comparison of regularly and seasonally occupied households," Sustain. Cities Soc., vol. 42, pp. 107-126, Oct. 2018.

[11] S. M. Hakimi, S. M. M. Tafreshi, and a. Kashefi, "Unit sizing of a standalone hybrid power system using particle swarm optimization (PSO)," in Proc. IEEE Int. Conf. Autom. Logist., 2007, Jinan, China, pp. 3107-3112.

[12] J. A. Domínguez-Navarro, R. Dufo-López, J. M. Yusta-Loyo, J. S. ArtalSevil, and J. L. Bernal-Agustín, "Design of an electric vehicle fastcharging station with integration of renewable energy and storage systems," Int. J. Electr. Power Energy Syst., vol. 105, pp. 46-58, Feb. 2019.

[13] M. A. M. Ramli, H. R. E. H. Bouchekara, and A. S. Alghamdi, "Optimal sizing of $\mathrm{PV} /$ wind/diesel hybrid microgrid system using multi-objective self-adaptive differential evolution algorithm," Renew. Energy, vol. 121, pp. 400-411, Jun. 2018.

[14] S. Mohseni and S.M. Moghaddas-Tafreshi, "Development of a multiagent system for optimal sizing of a commercial complex microgrid," arXiv preprint, arXiv:1811.12553, 2018.

[15] G. Mavrotas, K. Florios, and D. Vlachou, "Energy planning of a hospital using Mathematical Programming and Monte Carlo simulation for dealing with uncertainty in the economic parameters," Energy Convers. Manag., vol. 51, no. 4, pp. 722-731, Apr. 2010.

[16] R. Billinton and R. N. Allan, Reliability evaluation of engineering systems: concepts and techniques, Second. New York, NY: Plenum Press, 1992.

[17] K. P. Kumar and B. Saravanan, "Recent techniques to model uncertainties in power generation from renewable energy sources and loads in microgrids - A review," Renew. Sustain. Energy Rev., vol. 71, pp. 348358, May 2017.

[18] Y. Feng and S. M. Ryan, "Scenario construction and reduction applied to stochastic power generation expansion planning," Comput. Oper. Res., vol. 40, no. 1, pp. 9-23, Jan. 2013.

[19] S. Mohseni and S.M. Moghaddas-Tafreshi, "A multi-agent approach to optimal sizing of a combined heating and power microgrid," arXiv preprint, arXiv:1812.11076, 2018. 